\title{
Tissue Ischemia During Aortic Repair: The Point of View of the Perfusionist
}

\author{
De Simone Francesco, Tshiombo Gianbattista, \\ and Colombo Elisa
}

\subsection{Introduction}

Thoracoabdominal aortic surgery involves the cross-clamping of the descending thoracic aorta, which causes an abrupt reduction of the blood supply to both viscera and lower limbs. The reduction of blood flow induces the decrease of oxygen and substrates, which are essential for the Krebs cycle and the "respiratory chain." It also lowers the clearance of cellular catabolites, increases the probability of bleeding disorders, and prompts the release of inflammation mediators and free radicals [1-4].

The sudden exclusion of an extensive vascular district generates an immediate and significant increase of heart afterload and blood pressure. Furthermore, it is also associated with an increase in the cerebrospinal fluid (CSF) pressure [1-3, 5-7], which may determine a significant change in the perfusion pressure of the spinal cord (estimated as the gradient between blood pressure and CSF pressure) eventually leading to the collapse of its feeding vessels.

D. S. Francesco $(\bowtie)$

Department of Extracorporeal Circulation, IRCCS San Raffaele Scientific Institute, Milan, Italy e-mail: desimone.francesco@hsr.it

T. Gianbattista

Fondazione Cariplo, Milan, Italy

C. Elisa

Vascular Surgery Unit, IRCCS San Raffaele Scientific Institute, Milan, Italy
In the past, strategies such as expeditious surgery [8] or passive shunts [9] have been advocated to prevent these damages, however in more recent times, distal perfusion [7] has emerged as the method of choice.

In consideration of the foregoing, the teamwork of surgeons, anesthesiologists, perfusionists, nursing and allied healthcare professionals must be emphasized.

The aims of using distal perfusion during thoracic aortic cross-clamping are:

- Support of heart function, especially if already compromised [1, 2, 10-12]

- Reduction of end organ ischemia

- Control of proximal hypertension

\subsection{Different Methods for Distal Perfusion}

\subsubsection{Partial and Total Cardiopulmonary Bypass} (Fig. 15.1a, b)

During thoracoabdominal aortic surgery, distal perfusion may be obtained either by partial cardiopulmonary bypass (CPB) (Fig. 15.1a, b) [13] or left heart bypass (LHB) (Fig. 15.1c, d). The circuit for $\mathrm{CPB}$ consists of a venous cannula placed in a femoral vein or in the right atrium, a pump, an oxygenator, and an arterial cannula positioned in the 
a

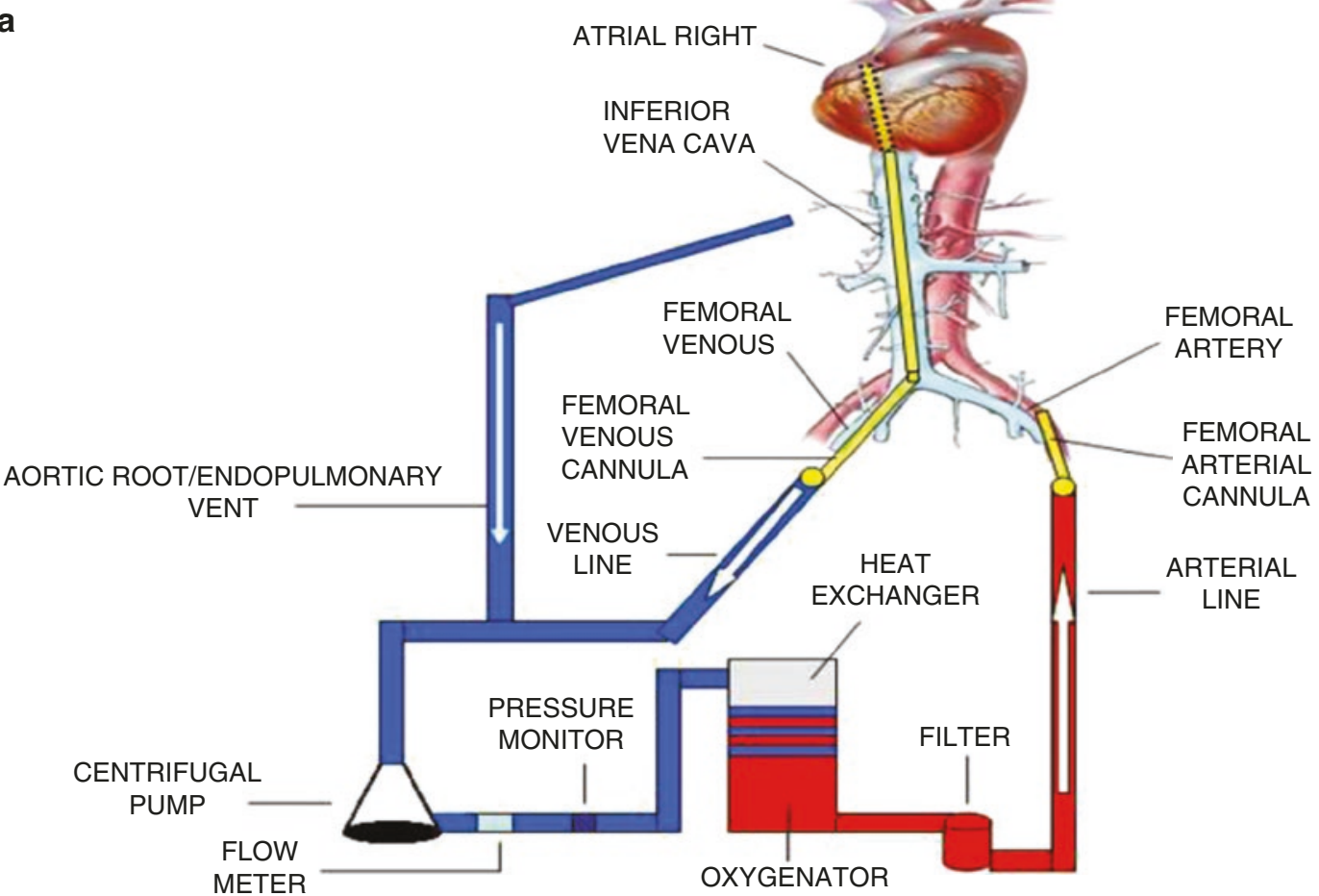

-The circuit for CPBP consists of a venous cannula placed in right atrium through the femoral vein, a pump, an oxygenator, and an arterial cannula positioned in femoral artery or in the distal aorta; a heat exchanger is also usually part of the CPBP circuit.-

b

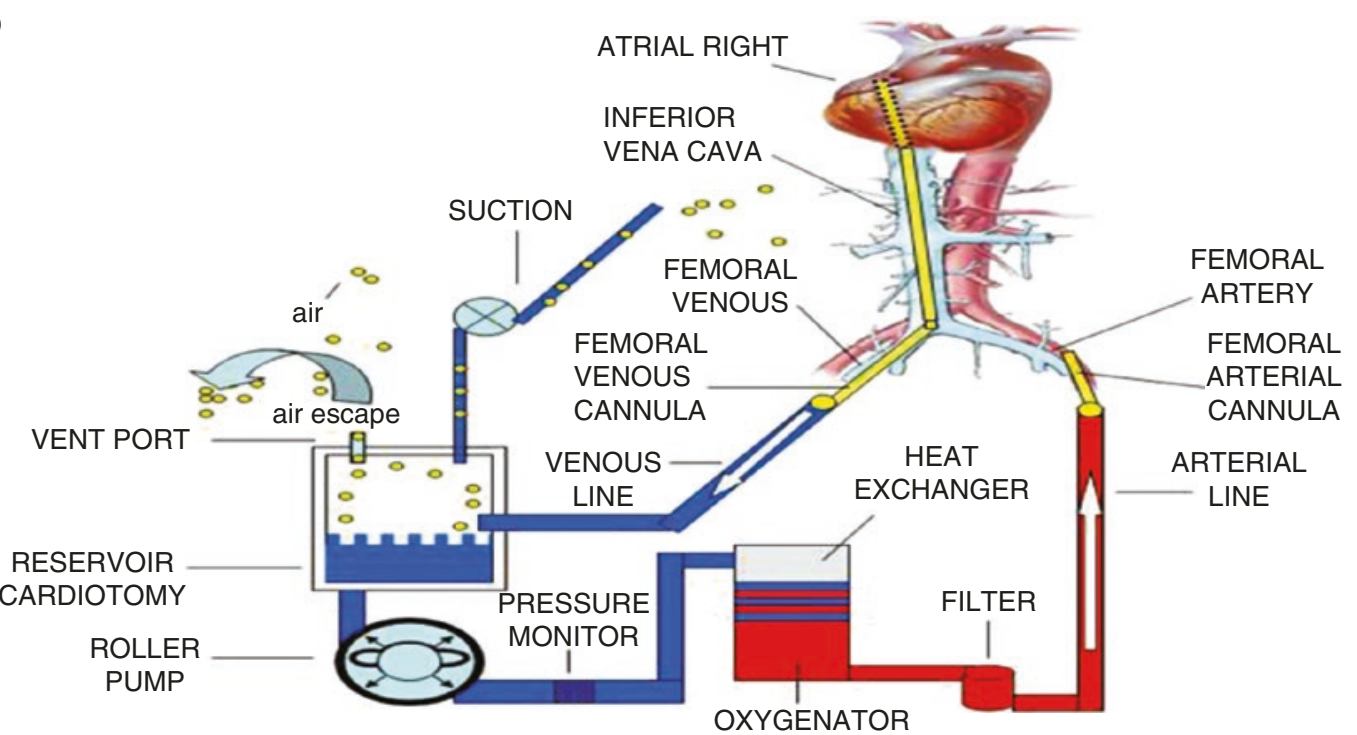

- A circuit for extracorporeal circulation is defined as "closed" when does not involve a contact between air and blood, and "open" when an air/blood interface is present.-

- "Open" systems, include a reservoir for venous blood and blood aspirated from the operative field.-

Fig. 15.1 (a) CPBP closed circuit. (b) CPBP open circuit. (c) Left bypass (LBP). (d) Left heart bypass (LHBP) 


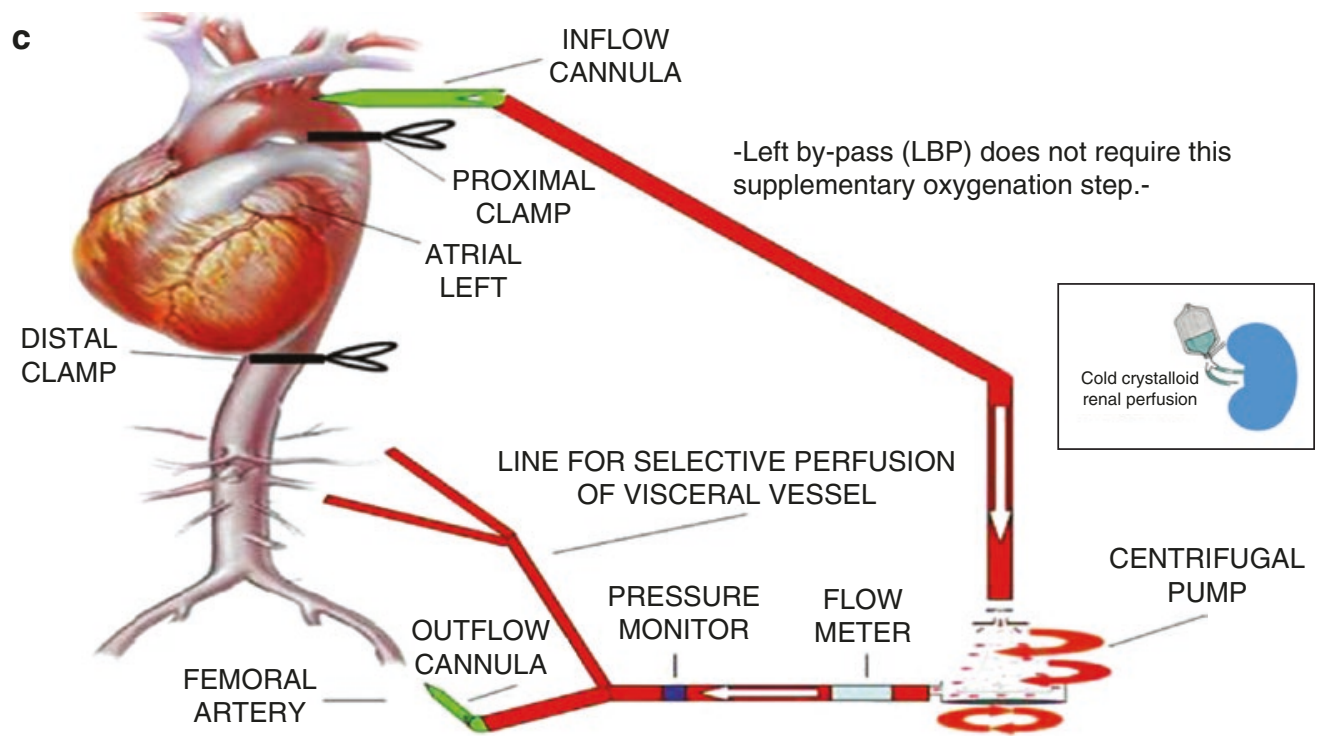

-When a LBP is used, the circuit is composed by an inflow cannula placed in the thoracic aorta proximal to the clamp, a pump, and an outflow cannula placed in the distal aorta or in the femoral artery.-

-A line for selective perfusion of visceral vessels needs to be positioned along outflow line.-

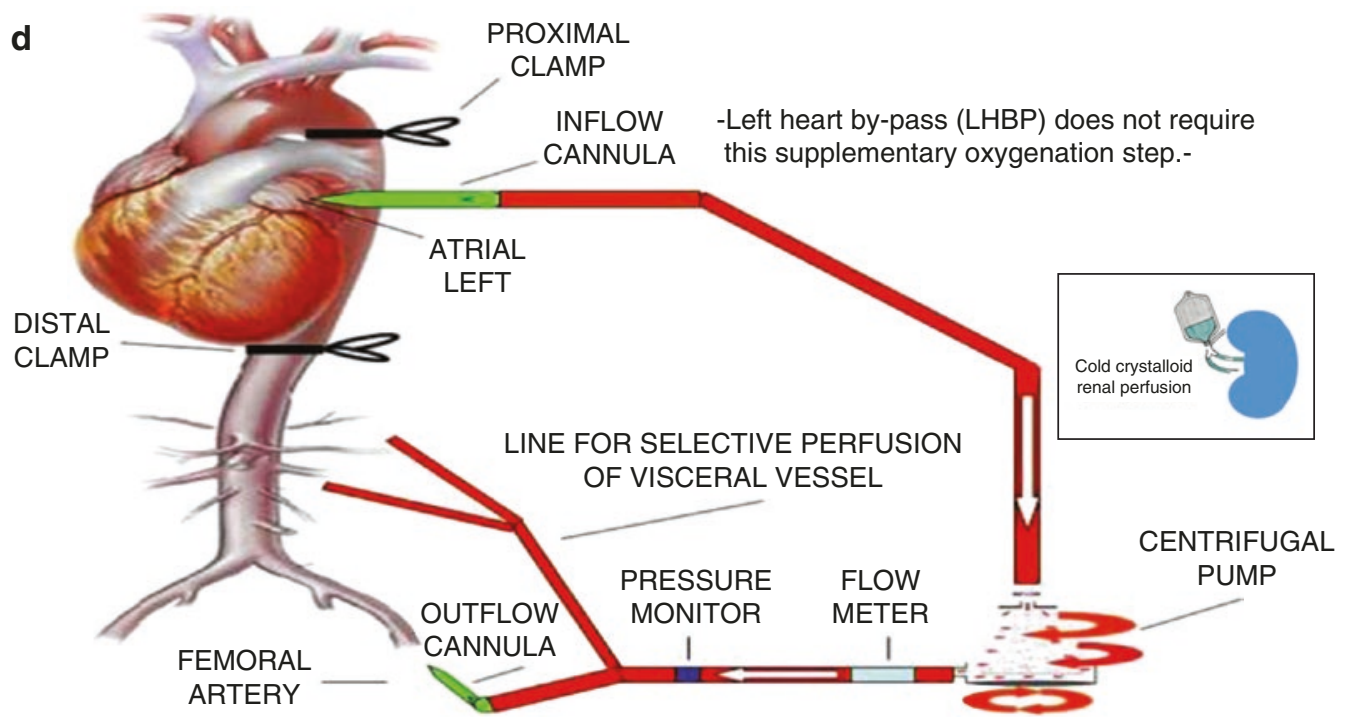

-When a LHBP is used, the circuit is composed by an inflow cannula placed in the pulmonary vein or left atrial appendage, a pump, and an outflow cannula placed in the distal aorta or in the femoral artery.-

-A line for selective perfusion of visceral vessels needs to be positioned along outflow line.-

Fig. 15.1 (continued) 
femoral artery or in the distal aorta [13]; usually, a heat exchanger is also part of the CPB circuit. When an LHB is used, the circuit is composed by an inflow cannula placed in the pulmonary vein or left atrial appendage (left heart bypass) (Fig. 15.1d), or in the thoracic aorta proximal to the clamp (left bypass) (Fig. 15.1c), a pump, and an outflow cannula placed in the distal aorta or in the femoral artery [7, 14]. The common goal of both systems is to ensure cardiac support and distal perfusion.

There are several differences between the two methods, each one having advantages and drawbacks (Table 15.1). The mechanism of partial CPB (Fig. 15.1a, b) works by taking a portion of blood from the venous district that is re-infused in the arterial compartment, bypassing the pulmonary circulation. This is why the blood needs to pass through an oxygenator; CPB requires full heparinization. Left bypasses do not require the oxygenation step and a lesser grade heparinization [7, 14].

A significant limitation of both systems is that they do not provide adequate perfusion in the upper body district in case of sudden and severe cardiac dysfunction. This occurs because the outflow cannula is positioned distally to the distal clamp [15]. Partial CPB, however (Fig. 15.1a, b), is more versatile than LHB (Fig. 15.1c, d) and in case of need, it can be converted into a total CPB by adding a second outflow branch with a cannula placed proximally to the aortic clamp.

Total CPB (an open system) (Fig. 15.1b) also offers the possibility to induce (profuse) hypothermia and perform a circulatory arrest. For more extensive operations, hypothermic circulatory arrest can be performed on full cardiopulmonary bypass by reducing the body temperature down to $16{ }^{\circ} \mathrm{C}$ to $18{ }^{\circ} \mathrm{C}$. This procedure protects the distal organs during ischemia, even though it is complicated by coagulopathy and the increased complexity of hypothermic cardiac arrest [16].

Total CPB with an interval of hypothermic circulatory arrest is widely used for surgeries on the ascending aorta and aortic arch, but less commonly exploited for operations on the thoracoabdominal aorta. Nevertheless, in these settings it offers several advantages since it requires only minimal dissection of the periaortic tissues, eliminates the need for proximal and sequential aortic
Table 15.1 Comparison between different extracorporeal circulation techniques

\begin{tabular}{|c|c|c|}
\hline Method & Pros & Cons \\
\hline $\begin{array}{l}\text { LHB- } \\
\text { CPB }\end{array}$ & $\begin{array}{l}\text { - Cardiac support } \\
\text { - Proximal arterial } \\
\text { pressure control } \\
\text { - Distal perfusion }\end{array}$ & $\begin{array}{l}\text { - Heparinization } \\
\text { needed } \\
\text { - Coagulation } \\
\text { cascade } \\
\text { - Inflammatory } \\
\text { response }\end{array}$ \\
\hline LHB & - Low heparin doses & $\begin{array}{l}\text { - Cannot replace } \\
\text { heart function in } \\
\text { case of failure } \\
\text { - Hemotransfusion } \\
\text { mandatory in } \\
\text { case of massive } \\
\text { bleeding }\end{array}$ \\
\hline $\begin{array}{l}\text { CPB } \\
\text { closed } \\
\text { circuit }\end{array}$ & $\begin{array}{l}\text { - Low heparin doses } \\
\text { - If needed it can be } \\
\text { switched into a total } \\
\text { CPB by adding a } \\
\text { second outflow } \\
\text { cannula proximally } \\
\text { to the aortic clamp }\end{array}$ & $\begin{array}{l}\text { - Oxygenator } \\
\text { needed } \\
\text { - More expensive } \\
\text { - High heparin } \\
\text { doses } \\
\text { - Need of } \\
\text { vasoactive drugs } \\
\text { - Hemotransfusion } \\
\text { mandatory in } \\
\text { case of massive } \\
\text { bleeding }\end{array}$ \\
\hline $\begin{array}{l}\text { CPB } \\
\text { open } \\
\text { circuit }\end{array}$ & $\begin{array}{l}\text { - No blood loss } \\
\text { - Less need of } \\
\text { crystalloids } \\
\text { supplementation } \\
\text { - Less need of plasma } \\
\text { and platelets } \\
\text { infusion } \\
\text { - Better management } \\
\text { of VF or congestive } \\
\text { heart failure } \\
\text { - May be used to } \\
\text { induce DHCA }\end{array}$ & $\begin{array}{l}\text { - High heparin } \\
\text { doses } \\
\text { - More expensive } \\
\text { - Need of venous } \\
\text { reservoir and } \\
\text { aspirators } \\
\text { - More difficult } \\
\text { hemostasis after } \\
\text { protamine } \\
\text { administration }\end{array}$ \\
\hline
\end{tabular}

clamping, and provides easy access to the distal aortic arch. It also offers a bloodless and quiet surgical field when the circulation is arrested and allows the recollection into the perfusion circuit of all blood lost [17].

Systemic heparinization is a fundamental factor, especially upon extensive surgical accesses or in emergency cases $[18,19]$. The need for heparinization is not particularly influenced by the circuit type used (CPB or LBP) (Fig. 15.1ad), but rather by the use of a "closed" (Fig. 15.1b) [13, 18-20] or "open" circuit. A closed extracorporeal circulation system does not involve a contact between air and blood, whereas it is defined 
"open" when an air/blood interface is present. Provided that a sufficiently high flow is maintained in order to prevent platelet aggregation in the circuit, a "closed" system requires only a mild anticoagulation [13] (less than $100 \mathrm{u} / \mathrm{kg}$ ) [1, 2, 20]. "Open" systems require total systemic anticoagulation, with the goal of maintaining the ACT $>480 \mathrm{~s}$, because they include a reservoir for venous blood and blood aspirated from the operative field implying contact between air and blood.

\subsubsection{Left Bypass and Left Heart Bypass (Fig. 15.1C, d)}

In our institution, the preferred method of circulatory assistance during thoracoabdominal aortic surgery is LBP (Fig. 15.1c, d). CPB (Fig. 15.1b), on the other hand, is used in selected cases (for instance, when the surgeon is not sure about the safety of proximal cross-clamping and circulatory arrest may be needed to perform an open proximal anastomosis).

In an LHB or LPB (Fig. 15.1c, d) setup, the heart perfuses everything proximal to the proximal aortic clamp (head and upper limbs). The patient must have both an adequate cardiac function to tolerate the clamping of the descending aorta and the consequent swings in the afterload, as well as good lung function to tolerate singlelung ventilation during a thoracotomy. Generally, the pump used for LBP (Fig. 15.1c, d) is centrifugal [16]: it consists of a bell in which a number of coaxial cones or fins are housed. The bell rests on a rotational magnet, which confers blood velocity with its fast rotary motion. The forced vortex principle $(f=m \times a)$ applies to very high fluid velocity: a central area with negative pressure (inflow zone) and a peripheral area with positive pressure (outflow zone) are generated within the bell. The kinetic energy produced by the pump and passed on the blood can overcome the resistance found throughout the circuit. The pump flow is directly proportional to the velocity, and inversely proportional to the resistance. A flowmeter is required because the outflow of the pump changes with the afterload at constant speed of the magnet.
As opposed to roller pumps (positive displacement pumps), centrifugal pumps cannot develop pressures that could be dangerous by breaking the circuit or damaging the vessels. Air embolism is a fairly rare complication, because the lack of mass prevents acceleration.

The main advantage is that this simple circuit can tolerate a lower activated clotting time (ACT) and in some cases no heparin at all [2]. Once LHB or LPB (Fig. 15.1c, d) is established the patient's temperature can be lowered to 32-33 ${ }^{\circ} \mathrm{C}$. This is because mild hypothermia decreases the risk of hypothermia-induced ventricular fibrillation. Arterial pressure is monitored proximal to the aortic clamp through a radial arterial line and distal to the clamp through a femoral arterial line [2].

Nevertheless, there are major disadvantages. One is the lack of reservoir, which, in the eventuality of massive blood loss does not allow the supply of blood directly to the patient, as it might be in a full CPB (Fig. 15.1b) setup. Then, it is not feasible to conversion to complete $\mathrm{CPB}$ (Fig. 15.1b) if the heart fibrillates or in case oxygenation on single-lung ventilation becomes problematic.

\subsubsection{Distal Perfusion}

The criteria for optimal distal perfusion during aortic cross-clamping are still debated. Some authors propose flows greater than $40 \mathrm{~mL} / \mathrm{kg} / \mathrm{min}$ [21], or flows ranging from 1.5 to $3 \mathrm{~L} / \mathrm{min}$ [2225] with a mean femoral artery pressure of $70 \mathrm{mmHg}[1,2,13,21-24]$. Others advise the use of motor evoked potentials meps.

Diuresis is a practical means for the evaluation of perfusion [26], although the non-pulsatile flow from the pump, the preoperative renal impairment, and hypo-perfusion may play a role in the etiology of anuria [27].

Other criteria for regulation of the pump depend on the area to perfuse (i.e., one or both lower limbs, kidneys, splanchnic vessels, etc.), on the relationship between the pressures developed by the heart (radial pressure), those developed by the pump (femoral pressure) and ventricular 
volumes monitored through trans-esophageal echocardiographic (tee) examination $[1,2,10,12]$. As far as distal perfusion is concerned, information on both pressure and flow is available, however concerning the proximal district, only blood pressure can be monitored, thus providing incomplete information on the real perfusion of organs such as the heart and the brain. Furthermore, due to a plethora of variables such as of the position of the patient on the surgical table, the pressure of the retractors and single-lung mechanical ventilation, the interpretation of parameters such as difficult and the importance of tee monitoring during aortic cross-clamping may not be overemphasized, especially in cardiopathic patients $[1,2,10,12]$.

\subsubsection{Components}

The basic circuit for LBP (Fig. 15.1c) or LHB (Fig. 15.1d) includes an inflow cannula, a pump, and an outflow cannula. A heat exchanger may be included to allow a better management of the patient's temperature by actively cooling organs. Consequently, it reduces the organs' metabolic rate $[2,21,28]$ before clamping and re-warming. However, hypothermia does not come without risks, which include cardiac arrhythmias [15] and coagulopathy $[26,27]$.

In our clinical practice, the heat exchanger is not used, since the patient passively cools down to approximately $34{ }^{\circ} \mathrm{C}$ before cross-clamping and LBP (Fig. 15.1c) or LHB (Fig. 15.1d) is discontinued at the time of clamp removal. Afterwards, rewarming is facilitated by warm infusions and a heat-exchange mattress placed beneath the patient.

In the LBP (Fig. 15.1c) or LHB (Fig. 15.1d) circuit, an accessory pathway may be positioned on the inflow line, to allow recirculation of fluids during the priming. In this way, any air bubbles accidentally aspirated in the circuit are eliminated and rapid re-infusion is facilitated. The blood aspirated from the operative field must pass through a cell-saver system and may then be re-infused through the accessory line mentioned above. The immediate restoration of blood volume is fundamental to maintaining stable hemodynamics.
Furthermore, adequate tissue perfusion is based on oxygen availability. Therefore, the preservation of all parameters that guarantee good oxygen supply to tissues, namely a good arterial oxygen saturation and a good level of hemoglobin, is fundamental. This is particularly true in situations where blood flow may not be optimal. The immediate re-infusion of autologous blood collected in the cell-saver also reduces the need of homologous transfusions, which may carry the risk of pulmonary damage [29] and others.

During the surgical anastomosis of the visceral vessels, abdominal organ perfusion may be continued by selective catheterization of the individual arteries. The line for selective perfusion of visceral vessels is to be positioned along the outflow one. Double lumen occlusion/perfusion 9-french catheters are used to cannulate the individual vessels [21, $25,30,31]$. Flows of $200-300 \mathrm{~mL} / \mathrm{min}$ for each catheter [32] maintain a visceral perfusion pressure of about $70 \mathrm{mmHg}$ [21, 31] (Fig. 15.1c, d).

Recent studies [33] have shown that perfusion of the renal arteries with cold crystalloid solutions is equally effective to protect the kidneys. In our practice, Custodiol ${ }^{\circledR}$ solution at $4{ }^{\circ} \mathrm{C}$ is used for renal perfusion, while the celiac and superior mesenteric arteries are perfused with warm blood from the pump (Fig. 15.1c, d).

\subsubsection{Priming of the Circuit}

The solutions used for priming vary according to the protocols employed in different centers. Generally, the priming of the circuit is done with $400 \mathrm{~mL}$ of crystalloid or colloid solutions, but in special cases (i.e., patients with anemia, or dialysis, etc.), the patient's autologous blood can be exploited. Technically, after the connection of the circuit with the inflow cannula and before the connection to the outflow cannula, it is possible to replace the priming with blood drawn from inflow cannula. When blood has substituted to the crystalloid solution in the circuit, the connection with the inflow cannula is completed. This maneuver induces some degree of hypovolemia that may result in a short period hemodynamic instability, which 


\section{Law of Laplace}

stress of wall

parity end diastolic aortic pressure (es. $50 \mathrm{mmHg}$ ) the wall's tension that ventricle must develop to open the aortic valve is low in normal preload ( $\mathrm{r} 2)$ and increase in high preload ( $\mathrm{r} 4)$

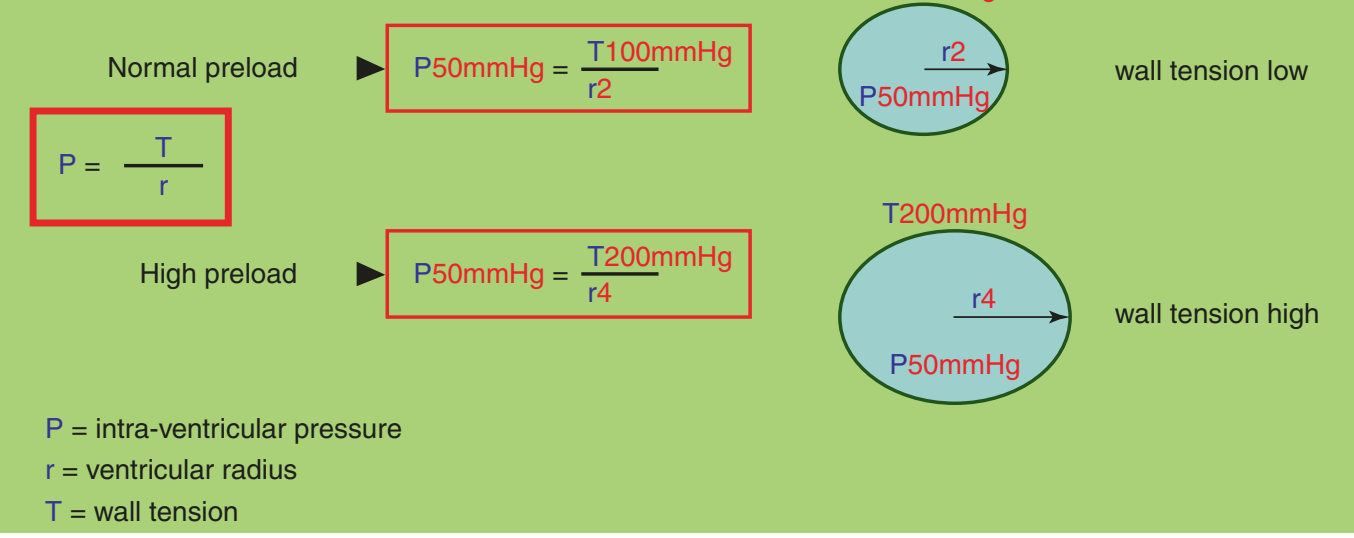

Fig. 15.2 The left ventricular afterload is determined also by the dimension of ventricle in the initial phase of iso-volumetric contraction

can be controlled pharmacologically or with the administration of homologous blood.

\subsubsection{Proximal Cannulation/Cardiac Support}

During the beginning of isovolumetric contraction, the afterload is influenced by the systemic resistance, related to aortic pressure, and by the preload, determined by ventricular end-diastolic volume. At the aortic cross-clamping, LBP (Fig. 15.1c) or LHB (Fig. 15.1d) supports heart function by reducing cardiac work. The inflow cannula can be positioned in the left atrium (LHB) (Fig. 15.1d) or in the thoracic aorta proximal to the clamp (LBP) (Fig. 15.1c).

\subsubsection{Inflow Cannula Positioned in the Left Atrium (LHB)}

(Fig. 15.1d)

The left ventricular afterload is determined by the dimension of ventricle in the initial phase of isovolumetric contraction; (Laplace $t=p r$ ) in other words the wall tension $(t)$ that the heart will need to develop to increase intra-ventricular pressure will be proportional to preload stand for the left ventricular radius $(r)$. (Fig. 15.2)

Aortic cross-clamping excludes much of systemic circulation, thus the direct effect is an increase of ventricular radius $(r)$ and the wall's tension that ventricle must develop to open the aortic valve (at the same end-diastolic aortic pressure) will be greater.

After aortic clamping, if the inflow cannula is positioned in the left atrium (left heart bypass) (Fig. 15.1d) drainage is made possible, the ventricular radius does not increase and the wall tension that the ventricle must develop to open the aortic valve will not be as high. Therefore, this placement benefits the heart by reducing the left ventricular volume (LVEDP) and oxygen consumption during isovolumetric contraction, by improving coronary perfusion and reducing pulmonary congestion. When the pulmonary stasis is reduced, both gas exchange and right ventricular function are also improved [1, 2, 6, 7, 34]. 
With atrial cannulation, care must be taken to avoid the aspiration of air from the outside, that would result in systemic embolism. From a surgical point of view, the cannulation of the left atrium may be obtained from either the left atrial appendage or the pulmonary vein. In our clinical practice, the left superior pulmonary vein is favored (Fig. 15.1d).

The surgeon has to be precise in positioning the left atrium cannula. A misplacement of the latter may result in the injection into the circuit of partially oxygenated blood coming in part from the nonventilated lung. This may be masked by a correct blood oxygen saturation measured in the upper body district.

\subsubsection{Inflow Cannula Positioned in the Thoracic Aorta (LBP)} (Fig. 15.1c)

The positioning of the inflow cannula in the thoracic aorta proximally to the clamp allows a smaller increase in afterload and peripheral resistance (SVR) induced by aortic cross-clamping, the consequence is a reduction of work for the left ventricle during isovolumetric contraction, in fact the ventricle does not need to increase the intracavitary pressure $(p)$ because the resistance against which it must work to open the aortic valve is not as high. Being the wall's tension $(t)$ directly proportional to the intra-ventricular pressure that the ventricle must develop, when the ventricular wall's tension is moderate the ventricular pressure will be moderate too, as a consequence the myocardial oxygen consumption $(\mathrm{MvO} 2)$ will be reduced [2].

This cannulation placement is particularly useful for patients with aortic or mitral insufficiency, because the afterload reduction determines the decrease of trans-valvular regurgitation.

\subsubsection{Distal Cannulation}

LBP (Fig. 15.1c) or LHB (Fig. 15.1d) requires relatively low flows, thus it allows the use of small diameter cannulas (i.e., 14-16-18 fr.). This represents an advantage especially when the inflow cannula is placed in the femoral artery. The cannula is secured with a purse-string suture and the artery is not occluded allowing distal perfusion of the ipsilateral limb. If the aortic crossclamping time is prolonged, this strategy avoids ischemia/reperfusion damage, which may also be associated with metabolic acidosis and renal failure [35, 36] (Fig. 15.3).

\subsection{Biocompatibility}

\subsubsection{Activation of Biological Circuits (Fig. 15.4)}

Blood has a very complex structure, consisting mainly of corpuscles (erythrocytes, leukocytes, and platelets) and plasma proteins. During bypass, blood is circulated via mechanical pump and homeostasis is impaired; intravascular pressures fluctuate outside normal ranges, fluid shifts occur with the extracellular compartment, and capillary permeability increases.

Direct injury to blood elements is produced by contact with synthetic (non-endothelial) surfaces, shear stress, turbulence, cavitation, and osmotic forces. Surface electrical charges are important in the repulsion or attraction of blood elements. The presence or absence of specific chemical groups, the surface tension, wettability of the polymer, and the presence of fillers and plasticizer are all relevant as well. Moreover, the activation of mediators triggers a series of reactions that involve the coagulation cascade, fibrinolysis, the $\mathrm{k}$ system, complement, and cytokines. When blood comes into contact with the synthetic materials of the various components of the extracorporeal circulation circuit, plasma proteins (albumin, gamma globulins, fibrinogen) immediately adsorb onto the circuit. This mechanism is determined by the material surface properties, being the plastic hydrophobic and with a high interfacial energy. The protein that initially adheres to the circuit walls is fibrinogen, which causes the adhesion of platelets mediated by the exposure of its hydrophobic residues that interact with the platelets GPIIb and GPIIIa receptors. Slow blood flow 


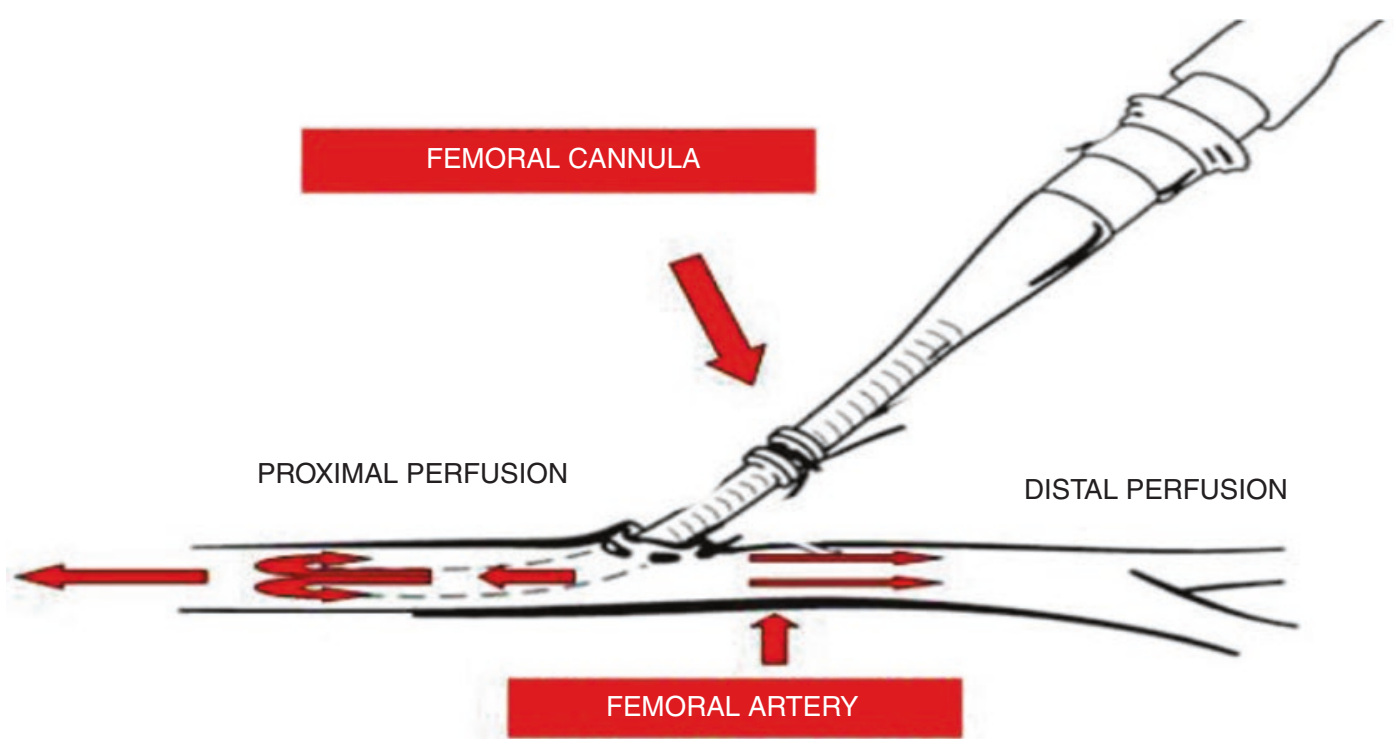

Fig. 15.3 The cannula is secured with a purse-string suture and the artery is not occluded allowing distal perfusion of the ipsilateral limb

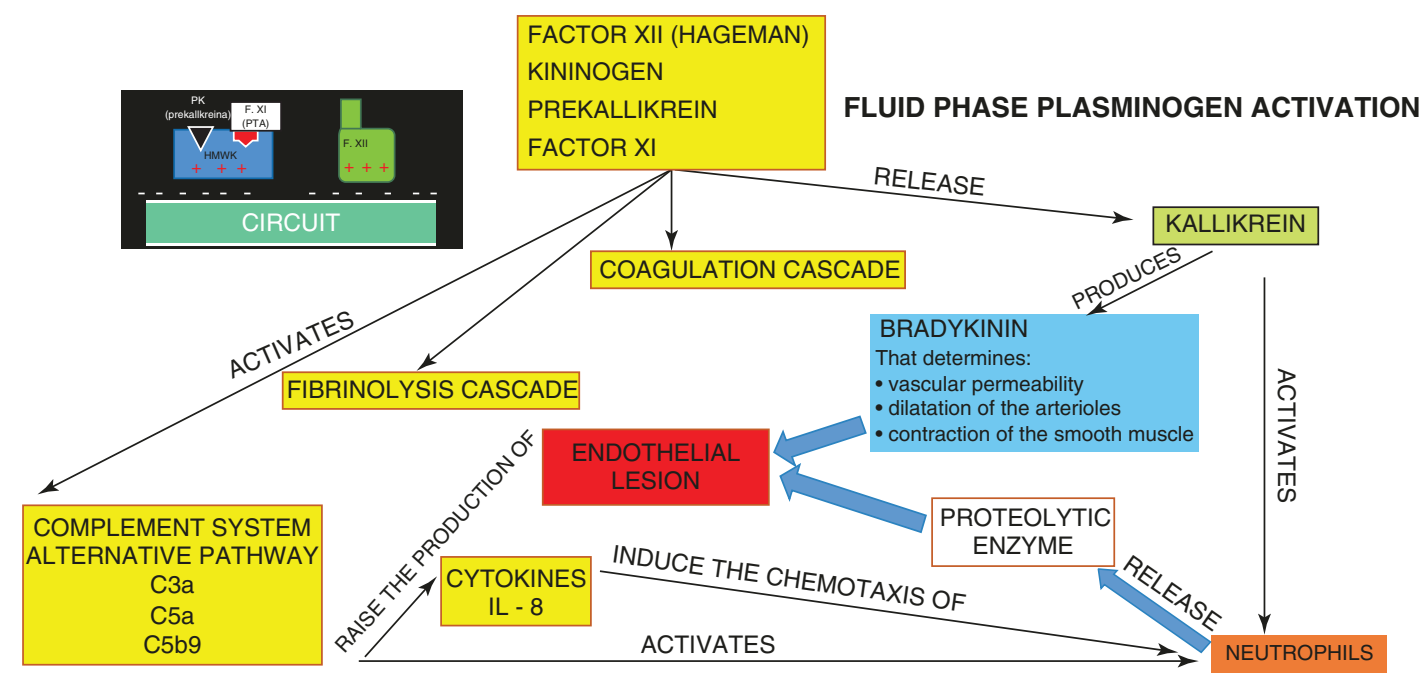

Fig. 15.4 Effects on blood and plasma components baited by extracorporeal bypass

may significantly increase the binding between platelets and foreign materials, this may happen, for instance, in the oxygenator meshes, through heat exchangers and in the filters present along the circuit, especially under hypothermic conditions. Adhesion, aggregation, and subsequent activation of platelets cause the release of granules and potent chemical inflammation mediators
( $\beta$-thromboglobulin, platelet factor 4 [PF4], etc.), which are an important determinants of postoperative coagulopathy.

By means of the intrinsic and extrinsic coagulation systems, extracorporeal circulation activates factor $\mathrm{X}$, which in turn triggers the common coagulation pathway that leads to clot formation. To avoid clotting, which may be fatal, heparin 
must be administered. In fact, by binding to antithrombin III, heparin accelerates its function through the inhibition of coagulation factors such as factor $\mathrm{X}$ and thrombin. Due to the contact of blood with the non-endothelial surfaces, immediately after starting extracorporeal circulation, the Hageman factor (factor XII) is activated. This factor acts on both the coagulation cascade, and fibrinolysis, the plasmin can further activate prekallikrein, the complement system and again the Hageman factor (Fig. 15.4).

The cascade of kallikrein determines the production of bradykinin, as well as the activation of other mediators, such as the Hageman XII factor or plasminogen [37]. Other factors that were previously underestimated, namely hemodilution, blood contact with air, complex heparinprotamine, etc., may contribute to the activation of the blood components [38].

\subsubsection{Metabolic Acid-Base Balance}

Metabolic acidosis is associated with $\mathrm{CPB}$, and the management of this complication must be aggressive. However, its pathogenesis is poorly understood because the traditional methods of examining acid-base changes (i.e., Anion Gap Analysis) are lacking in this setting. The effect of the composition of pump priming solution on serum lactate concentration has been studied, and it has been demonstrated that the use of a solution with no lactate significantly limits the increase in serum concentrations of this chemical in the postoperative period.

Metabolic acidosis also tends to develop during $\mathrm{CPB}$, even when apparently adequate flow rates are used [39]. This is probably related to the uneven distribution of flow during CPB, with the consequent development of hypoperfused areas that release lactic acid. Usually, the resultant metabolic acidosis is not severe, and the concentration of lactic acid rarely exceeds $5 \mathrm{mmol} / \mathrm{L}$ [3].

Anesthesiologists' expertise is fundamental to face the following responses to cardiopulmonary bypasses techniques: whole-body (nonspecific: neutrophil activation, platelet response) complement activation inflammatory response to use of a pump-oxygenator, humoral response (kallikrein-bradykinin activation, fibrinolytic cascade, coagulation cascade, arachidonic acid cascade, cytokines), cellular response, metabolic response.

\subsubsection{Distribution of Blood Flow}

\subsubsection{Brain}

During euthermic and moderately hypothermic CPB in adults and elderly patients, cerebral blood flow is not importantly influenced by variations of mean arterial blood pressure [21, 22]. This is similar to what happens to normal awake adult humans, in whom cerebral blood flow does not vary significantly with variations of about $60-150 \mathrm{mmHg}$ in mean arterial blood pressure.

Cerebral blood flow during CPB is affected by arterial carbon dioxide pressure (PaCO2). Hypercarbia increases cerebral blood flow and hypocarbia decreases it [23-25]. Usually, when mean blood pressure is lower than $40 \mathrm{mmHg}$ for more than a few minutes during re-warming, anesthesiologists should elevate arterial blood pressure.

Blood flow to the skin is severely reduced during non-pulsatile CPB. The off-pump cardiac surgery patients had less severe, but statistically significant, microcirculatory alterations immediately postoperatively. These alterations improved slightly but persisted after 24 h. Thus, microvascular perfusion presented similar alterations in CPB and off-pump patients 6-24 h after admission to the intensive care unit.

Veins constrict during CPB by an increase in venous tone that may persist for some hours afterward. The mechanism of this vasoconstriction has not been determined with certainty, even if high levels of circulating catecholamines probably play an important role.

\subsubsection{Kidney}

Ischemia stops blood supply to the glomerulus and post glomerular vessels, if it persists more than 10 min acute tubular necrosis, which is the most frequent form of acute renal failure (70\%), may result.

Postoperative dialysis-dependent renal failure is associated with a 50-60\% mortality risk; thus, 
the preservation of renal function and the avoidance of dialysis reduce mortality risk and improve long-term survival [30, 40].

From the cellular point of view, the whole tubule can undergo necrosis with the terminal portions of the proximal tubule and the ascending branch of the Henle loop, that are seats of important metabolic processes, having a higher tendency. The cells of these districts receive nourishment from peritubular capillaries. A reduction in perfusion, therefore a drop in the oxygen supply to these cells, leads to the loss of cytoskeletal polymerization with consequent loss of the brush-border and cell polarity. The result is a dislocation of the $\mathrm{Na}^{+} / \mathrm{K}^{+}$pump with loss of the resorption capacity of the tubular solutes.

The brush-like orifice disappears, the cell-cell connections are no longer stable, and the cell falls into the tubular lumen, where cellular debris adheres to a protein substance produced by the tubule (Tamm-Horsfall glycoprotein). The outcomes are cylinders occluding the lumen and preventing the passage of urine from the occluded tubule.

The open spaces on the lateral-lateral slope favor the back diffusion of solutes and luminal water (pre-urine) towards the peritubular circulation, where they are reabsorbed and recirculated. The reflux of urinary material in the interstitial space causes acute inflammation with edema.

Perfusion at higher pressures with moderate or deep hypothermia (hypothermic arrest or cold renal perfusion decreasing renal temperature to $<20{ }^{\circ} \mathrm{C}$ during occlusion) appears to provide effective renal protection, with dialysis rates of $3 \%$ or less in most reports whether cold blood or crystalloid is used [31-34] (Fig. 15.1c, d).

\subsubsection{Viscera}

The physiology of splanchnic circulation is complex and not fully understood. Different intrinsic (metabolic and myogenic) and extrinsic (neural and humoral) systems regulating the intestinal blood flow interact with each other. Self-regulation of blood flow pressure, reactive hyperemia, and hypoxic vasodilation are considered intrinsic control mechanisms and are responsible for instantaneous changes in blood flow in the splanchnic district. According to the metabolic theory, the distribution of oxygen has a greater influence than blood flow on variations in the splanchnic circulation. Therefore, an imbalance between oxygen supply and tissue demand will result in a greater concentration of local metabolites such as hydrogen, potassium, carbon dioxide, and adenosine, which will cause vasodilatation and hyperemia. In accordance with the myogenic theory, the voltage receptors of the arterioles regulate the vessel resistance in relation to the transmural pressure. The extrinsic neural factors of regulation of the splanchnic circulation include the $\alpha$-adrenergic vasoconstrictive fibers, their activation results in vasoconstriction of the small vessels and a reduction of the mesenteric blood flow.

After 35-40 minutes of complete normothermic exclusion from the circulation, liver function is restored without consequences [35]. The time lapse increases to 60 minutes in the case of mild hypothermia $\left(20-22{ }^{\circ} \mathrm{C}\right.$ ), after which liver function resumes without structural or functional abnormalities [36].

Intestinal hypothermia experimentally induced in rats by maintaining temperature tissue between 15 and $20^{\circ} \mathrm{C}$ has been shown to protect intestinal mucosa from damage associated with mesenteric ischemia. Although the mechanism remains not entirely known, protection is likely to be guaranteed by multiple factors: reduced consumption of oxygen and ATP, reduced formation of ROS, and reduced activation of the Nf-kb. [41].

\subsubsection{Spinal Cord}

Hypothermia provides important protection of the spinal cord during ischemic intervals produced by aortic clamping. In a clinical study of hypothermic CPB and circulatory arrest (mean interval of arrest, $38 \mathrm{~min}$; range, 8-62 min) among the 109 survivors with thoracoabdominal aortic disease, early paraplegia occurred in 1 of 36 with Crawford extent I, 0 of 42 with extent II, and 2 of 31 with extent III disease [42].

During surgeries on the descending thoracic and thoracoabdominal aorta on 161 patients, the prevalence of paraplegia or paresis from spinal cord ischemia remained constant and less than $3.5 \%$ for ischemic (but hypothermic) intervals of up to $138 \mathrm{~min}$ [39]. 


\subsubsection{Biocompatibility of Materials}

Modern extracorporeal circulation circuits have an internal biopolymers coating (usually made of heparin or phosphorylcholine) [20,42]. The coatings mimic the endothelial lining and so reduced the requirement for anticoagulation and decrease the inflammatory response. Nevertheless, the inner surfaces of these circuits are far from complete biocompatibility and do not perfectly replicate the endothelium that is capable of preventing blood elements adhesion clot formation.

As soon as plasma proteins come in contact with the surface, they are adsorbed on to heparinized blood onto all non-endothelial cell surfaces to form a monolayer of bound proteins. For a specific protein, the amount adsorbed depends on its plasma concentration and the intrinsic activity of the artificial surface. The physical and chemical compositions of the polymer determine the proteins, not necessary the most abundant ones, which will likely adhere to that surface. Being every extracorporeal circuit made of a variety of different biomaterials, chemical, physical, and morphological properties of each polymer surface must be considered for the assessment of the adsorbed proteins, which compete for space on the biomaterial surface, are tightly packed, irreversibly bound, and immobile.

Fibrinogen is one of the main proteins adsorbed by most artificial surfaces (it adheres more selectively to hydrophobic rather than hydrophilic materials).

The surfaces constantly change their protein layer as blood flows. Moreover, as proteins adhere to the surface, they can undergo specific conformational changes and expose amino-acid receptor groups recognized by determined blood cells or plasma proteins such as factor XII, complement protein (C3a-C5a etc.), or platelets. Uncontrolled conformational changes of adsorbed protein vary among different biomaterial surfaces and change the reactivity of the adsorbed protein with cells and blood components. In contrast, endothelial cells selectively bind specific proteins using specialized receptors in response to cellular signals that regulate anticoagulants and pro-coagulants mediators. Different biomaterials vary in thrombogenicity, but all eventually simulate clot formation.

\subsection{Conclusions}

The precise isolation of the individual contributors to better results observed in thoracoabdominal aortic surgery in recent times [41] is not possible. The explanation is multifactorial. In fact, not only the nature of the disease and the characteristics of the operation challenge the integrity and the function of all organs systems, but also many factors contribute to the success or failure of the procedure. Overall, the improvement is likely to be related to the synergism of many small variations in the use of surgical and anesthetic techniques and technological novelties.

According to our experience, it is relevant to underline the substantial contribution of better materials and a more rational use of the existing extracorporeal circulation systems among the many technological improvements that have taken place.

\section{References}

1. Kahn RA, Stone ME. Anesthetic consideration for descending thoracic aortic aneurysm repair. Semin Cardiothorac Vasc Anesth. 2007;11(3):205-23.

2. Hessel EA. Techniques for descending thoracic aorta surgery. Semin Cardiothorac Vasc Anesth. 2001;5:293-320.

3. Schepens M, Dossche K. Introduction of adjuncts and their influence on changing results in 402 consecutive thoracoabdominal aortic aneurysm repairs. Eur $\mathbf{J}$ Cardiothorac Surg. 2004;25(5):701-7.

4. Pantin EJ, Cheung AT. Thoracic aortic disease. In: Kaplan JA, Reich DL, Lake CL, et al., editors. Kaplan's cardiac anesthesia. 5th ed. Philadelphia: Saunders Elsevier; 2006. p. 723-64.

5. Coselli JS, Lemaire SA. Descending and thoracoabdominal aortic aneurysms. In: Cohn LH, editor. Cardiac surgery in the adult. 3rd ed. New York: McGraw-Hill Medical; 2008. p. 1277-98.

6. Coselli JS. The use of left heart in the repair of thoracoabdominal aortic aneurysms: current techniques and results. Semin Thorac Cardiovasc Surg. 2003;15(4):326-32.

7. Crawford ES, Walker HS. Graft replacement of aneurysm in descending thoracic aorta: results without bypass or shunting. Surgery. 1981;89(1):73-85.

8. Murray GF, Young WG Jr. Thoracic aneurysmectomy utilizing direct left ventriculofemoral shunt (TDMACheparin) bypass. Ann Thorac Surg. 1976;21(1):26-9.

9. Fayad A, Sawchuk C. Transesophageal echocardiography in the management of left atrio-femoral during 
thoracoabdominal aortic aneurysm repair: a case report. Can J Anaesth. 2002;49(10):1081-3.

10. Suzuki S, Davis CA. Cardiac function predicts mortality following thoracoabdominal and descending thoracic aortic aneurysm repair. Eur. J Cardiothorac Surg. 2003;24(1):119-24.

11. Fayad A, Yang H. Acute diastolic dysfunction in thoracoabdominal aortic aneurysm surgery. Can J Anaesth. 2006;53(2):168-73.

12. Palombo D, Valenti D. Early experience with the minimal extracorporeal circulation system (MECC) during thoracoabdominal aortic aneurysm repair. Eur J Vasc Endovasc Surg. 2004;27(3):324-6.

13. Moore EE, Burch JM. Repair of the torn descending thoracic aorta using the centrifugal pump for partial left heart. Ann Surg. 2004;240(1):38-43.

14. Edwards JT, Brown DM. A versatile extracorporeal circuit for use during repair of descending and thoracoabdominal aortic aneurysms in high-risk patients with cardiac and/or pulmonary dysfunction: a novel approach to a significant perfusion management dilemma. J Extra Corpor Technol. 2004;36(3):245-9.

15. Ziganshin B, Elefteriades JA. Deep hypothermic circulatory arrest. Ann Cardiothorac Surg. 2013;2(3):303-15.

16. Kouchoukos NT. TAAA repair using hypothermic cardiopulmonary bypass and circulatory arrest. Ann Cardiothorac Surg. 2012;1(3):409-11.

17. Leach WR, Sundt TM. Oxygenator support for partial left-heart. Ann Thorac Surg. 2001;72(5):1770-1.

18. Downing SW, Cardarelli MG. Heparinless partial cardiopulmonary for the repair of aortic trauma. J Thorac Cardiovasc Surg. 2000;120(6):1104-9.

19. Tagarakis GI, Tsilimingas NB. Heparin-coated extracorporeal circulation systems in heart surgery. Recent Pat Cardiovasc Drug Discov. 2009;4(3):177-9.

20. Paparella D, Yau TM. Cardiopulmonary induced inflammation: pathophysiology and treatment. An update. Eur J Cardiothorac Surg. 2002;21(2):232-44.

21. Greeley WJ, Ungerleider RM, Kern FH, Brusino FG, Smith LR, Reves JG. Effects of cardiopulmonary bypass on cerebral blood flow in neonates, infants, and children. Circulation. 1989;80:I209.

22. Govier AV, Reves JG, McKay RD, Karp RB, Zorn GL, Morawetz RB, et al. Factors and their influence on regional cerebral blood flow during nonpulsatile cardiopulmonary bypass. Ann Thorac Surg. 1984;38:592.

23. Murkin JM, Farrar JK, Tweed WA, McKenzie KN, Cuirauden G. Cerebral autoregulation and flow/metabolic coupling during cardiopulmonary bypass: the influence of PaCO2. Anesth Analg. 1987;66:825.

24. Prough DS, Stump DA, Roy RC, Gravlee GP, Williams T, Mills SA, et al. Response of cerebral blood flow to changes in carbon dioxide tension during hypothermic cardiopulmonary bypass. Anesthesiology. 1986;64:576.

25. Ataka K, Okada M. Clinical study of optimal flow for temporary with centrifugal pump in surgical treatment of aneurysm of the descending thoracic aorta. Nippon Kyobu Geka Gakkai Zasshi. 1994;42(6):879-85.

26. Szwerc MF, Benckart DH. Recent clinical experience with left heart using a centrifugal pump for repair of traumatic aortic transection. Ann Surg. 1999;230(4):484-90.
27. Cimen B, et al. Pulmonary function tests, aerobic capacity, respiratory muscle strength and endurance of patients with rheumatoid arthritis. Clin Rheumatol. 2001;20:168-73.

28. Jacobs MJ, Mommertz G. Surgical repair of thoracoabdominal aortic aneurysms. J Cardiovasc Surg. 2007;48(1):49-58.

29. Cimen OB, et al. Pulmonary function tests, respiratory muscle strength, and endurance of patients with osteoporosis. South Med J. 2003;96:423-6.

30. Jacobs MJ, et al. Prevention of renal failure in patients undergoing thoracoabdominal aortic aneurysm repair. J Vasc Surg. 2004;40:1067-73. discussion 1073.

31. Kouchoukos NT, et al. Hypothermic bypass and circulatory arrest for operations on the descending thoracic and thoracoabdominal aorta. Ann Thorac Surg. 1995;60:67-76. discussion 76-77.

32. Fehrenbacher JW, et al. Optimal end-organ protection for thoracic and thoracoabdominal aortic aneurysm repair using deep hypothermic circulatory arrest. Ann Thorac Surg. 2007;83:1041-6.

33. Koksoy C, et al. Renal perfusion during thoracoabdominal aortic operations: cold crystalloid is superior to normothermic blood. Ann Thorac Surg. 2002;73:730-8.

34. Huguet C, Nordlinger B, Bloch P, Conard J. Tolerance of the human liver to prolonged normothermic ischemia. Arch Surg. 1978;113:1448.

35. Rittenhouse EA, Mohri H, Reichenbach DD, Merendino KA. Morphological alterations in vital organs after prolonged cardiac arrest at low body temperature. Ann Thorac Surg. 1972;13:564.

36. Hassoun HT, Kozar RA, Kone BC, Safi HJ, Moore FA. Intraischemic hypothermia differentially modulates oxidative stress proteins during mesenteric ischemia/reperfusion. Surgery. 2002;132:369-76.

37. Swaminathan M, Phillips-Bute BG. The association of lowest hematocrit during cardiopulmonary bypass with acute renal injury after coronary artery bypass surgery. Ann Thorac Surg. 2003;76(3):784-91.

38. De Somer F, Van Belleghem Y. Phosphorylcoline coating offers natural platelet preservation during cardiopulmonary bypass. Perfusion. 2002;17(1): 39-44.

39. Harris EA, Seelye ER, Barratt-Boyes BG. Respiratory and metabolic acid-base changes during cardiopulmonary bypass in man. Br Anesth. 1970;42:912.

40. Hanssen SJ, Derikx JP. Visceral injury and systemic inflammation in patients undergoing extracorporeal circulation during aortic surgery. Ann Surg. 2008;248(1):117-25.

41. Kouchoukos NT, Masetti P, Rokkas CK, Murphy SF, Blackstone EH. Safety and efficacy of hypothermic ardiopulmonary bypass and circulatory arrest for operations on the descending thoracic and thoracoabdominal aorta. Ann Thorac Surg. 2001;72:699.

42. Brusino FG, Reves JG, Smith LR, Prough DS, Stump DA, McIntyre RW. The effect of age on cerebral blood flow during hypothermic cardiopulmonary bypass. J Thorac Cardiovasc Surg. 1989;97:541. 